\title{
IoT-Lite: A Lightweight Semantic Model for the Internet of Things
}

\author{
Maria Bermudez-Edo \\ Software Engineering Department \\ University of Granada \\ Granada, Spain \\ Email: mbe@ugr.es
}

\author{
Tarek Elsaleh, Payam Barnaghi, Kerry Taylor \\ Institute for Communication Systems \\ University of Surrey \\ Guildford, United Kingdom \\ Email: \{t.elsaleh, p.barnaghi, k.taylor\}@surrey.ac.uk
}

\author{
Kerry Taylor \\ The Australian National University \\ Canberra, Australia \\ Email: kerry.taylor@anu.edu.au
}

\begin{abstract}
Over the past few years the semantics community has developed ontologies to describe concepts and relationships between different entities in various application domains, including Internet of Things (IoT) applications. A key problem is that most of the IoT related semantic descriptions are not as widely adopted as expected. One of the main concerns of users and developers is that semantic techniques increase the complexity and processing time and therefore they are unsuitable for dynamic and responsive environments such as the IoT. To address this concern, we propose IoT-Lite, an instantiation of the semantic sensor network (SSN) ontology to describe key IoT concepts allowing interoperability and discovery of sensory data in heterogeneous IoT platforms by a lightweight semantics. We propose 10 rules for good and scalable semantic model design and follow them to create IoT-Lite. We also demonstrate the scalability of IoT-Lite by providing some experimental analysis, and assess IoT-Lite against another solution in terms of round time trip (RTT) performance for query-response times.
\end{abstract}

Keywords-Internet of Things; Semantics; Linked Sensor Data; Knowledge Management

\section{INTRODUCTION}

With the growing development of machine-to-machine communications and IoT deployments, interoperability between different platforms has become a key issue in creating large scale IoT frameworks. Semantic technologies suggest a suitable approach for interoperability by sharing common vocabularies, and also enabling interoperable representation of inferred data. IoT testbed providers have recently started to add semantics to their frameworks allowing the creation of the semantic Sensor Web, which is an extension of the current Web in which information is given well-defined meaning, enabling machine-to-machine communications and interactions between objects, devices and people [10].

Semantics usually model the domain concepts in great detail. Although they can be applied for querying almost anything about objects, these complex models are often difficult to implement and use, especially by non-experts. They demand considerable processing resources and therefore they are considered unsuitable for constrained environments. Instead, IoT models should consider the constraints and dynamicity of the IoT environments, especially with the new trend towards integrating semantics in constrained devices such as M2M gateways or smartphones. At the same time, they need to model the relationships and concepts that represent and allow interoperability between IoT entities. Therefore, expressiveness versus complexity is a challenge.

It is important to note that semantic models are not end-products. They are normally only part of a solution and should be transparent to the end user. The semantic annotation models should be offered with effective methods, API's and tools to process the semantics in order to extract actionable information from raw data. Query methods, machine learning, reasoning and data analysis techniques should be able to effectively use these semantics. Semantic modelling is only the initial part of the whole design, and it has to take into account how the models will be used; how the annotated data will be indexed and queried with real-time data; and how to make the publication suitable for constrained environments and large scale deployments when applications often require low latency and processing time.

We propose IoT-Lite, a lightweight semantic model which is an instantiation of the Semantic Sensor Network (SSN) ontology [2](see Figure 1). IoT-Lite is the outcome of a research effort that focuses on discovery and seeks for the minimum concepts and relationships that can provide answers to most of the end user queries. We have focused on the typical queries for accessing the data in the IoT based on our experience in the challenge of analysing data for obtaining meaningful information for end-users. We find that we do not need full descriptions and complex relationships to satisfy user queries. Some of the most commonly used semantic models on the Web are simple models, such as Friend Of A Friend, (FOAF) ${ }^{1}$. Their simplicity encourages faster adoption by end users, as they do not imply complex annotations or do not require complex processing methods. Optimised models can also support providing faster responses to queries.

We also propose guidelines for developing scalable and reusable semantic models in the IoT. These guidelines leverage conventions followed by some semantic modelling designers such as the utilisation of the linked data approach.

IoT-Lite does not intend to be a full ontology for the IoT. Our aim is to create a core lightweight ontology that allows

\footnotetext{
${ }^{1}$ http://www.foaf-project.org/
} 


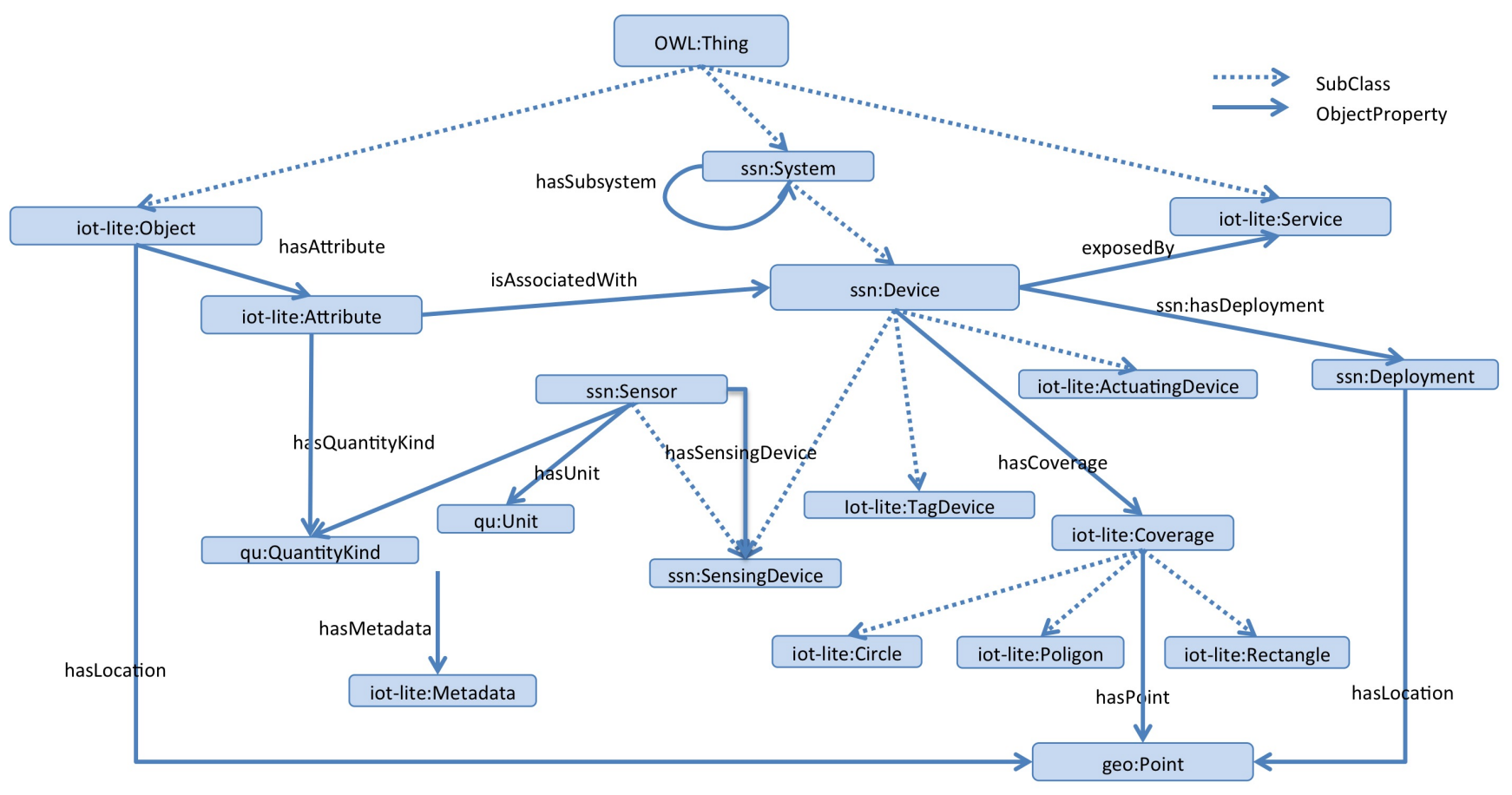

Figure 1: An overview of the proposed semantic model, IoT-Lite.

relatively fast annotation and processing time. IoT-Lite can be a core part of a semantic model in which, depending on the applications, different semantic modules can be added to provide additional domain and application specific concepts and relationships.

The remainder of the paper is organised as follows. Section II describes the related work. Section III introduces the 10 rules for good and scalable semantic model design and presents the proposed model, IoT-Lite, for representation of IoT elements. Section IV provides a use case scenario that illustrates the semantic annotation of a sensor in our model. Section V details an evaluation of the proposed model against a more detailed model and Section VI concludes the paper and describes the future work.

\section{RELATED WORK}

There are several semantic descriptions designed for the IoT domain. The SSN ontology [2] is one of the most significant and widespread models to describe sensors and IoT related concepts.

The SSN Ontology provides concepts describing sensors, such as outputs, observation value and feature of interest. However it is a detailed description, containing concepts and properties that enable flexible descriptions over a very wide range of applications, but including non-essential components for many use cases that can make the ontology heavy to query and process if it is used as it is.
The IoT-A model ${ }^{2}$ and IoT.est [14] are some of the projects that extend the SSN ontology to represent other IoT related concepts such as services and objects in addition to sensor devices. IoT-A provides an architectural base for further IoT projects (see Figure 2). The only implementation of a purely IoT-A semantic model known by the authors is described in [3]. The IoT-A model is overly complex for fast user adaptation and responsive environments. The IoT.est model extends the IoT-A model with extended service and test concepts.

The Open Geospatial Consortium (OGC), through its Sensor Web Enablement (SWE) group [1] has developed a set of standards to describe sensors and their data, for example, SensorML ${ }^{3}$, which is a language to describe sensors, and Observations and Measurements (O\&M). While SensorML provides important syntactic descriptions using XML, it lacks the expressibility provided by ontology languages such as OWL. SemSOS [4] has mapped the XML tags of O\&M into OWL concepts. However it represents only observations and not other IoT related concepts.

One of the ongoing works is OneM2M. OneM2M has published a report for home automation, and describes concepts and relationships [8]. Another initiative is the Spatial

\footnotetext{
${ }^{2}$ www.iot-a.eu/

${ }^{3}$ http://www.opengeospatial.org/standards/sensorml
} 


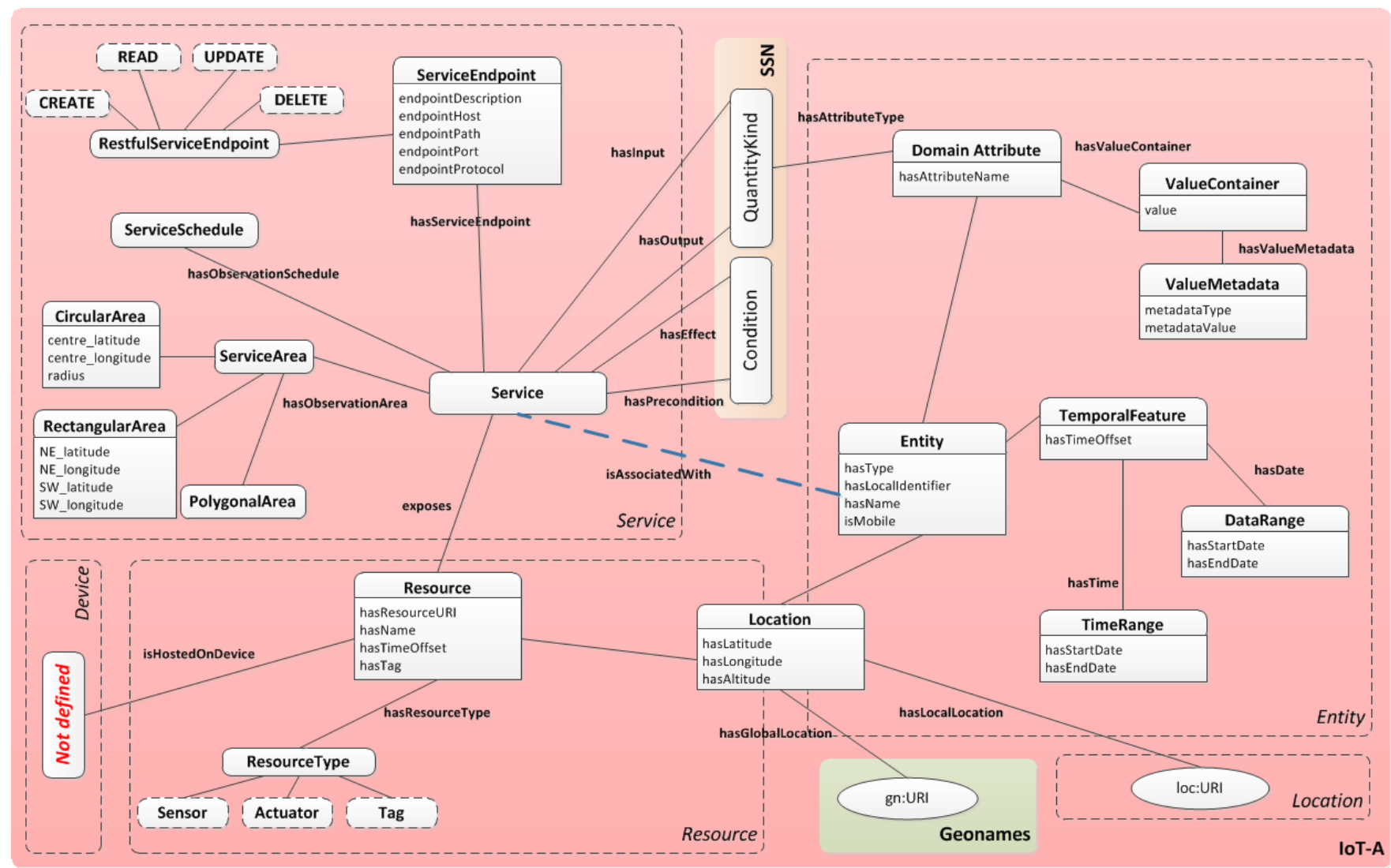

Figure 2: An example of a sensor annotated with the proposed IoT-Lite ontology.

Data on the Web Working Group ${ }^{4}$, a joint effort between the World Wide Web Consortium (W3C) and the Open Geospatial Consortium (OGC) that aims to standardise key ontologies for spatial, temporal and sensor data on the web [12]. Several projects also work on semantic descriptions for the IoT, such as FED4FIRE ${ }^{5}$ that currently has a semantic model focus on communications, VITAL ${ }^{6}$ for smart cities, CityPulse $^{7}$ with more focus on data and OpenIoT ${ }^{8}$, which is an instantiation of SSN.

Performance of ontologies for large data set have been addressed by different views, such as by redesign the data knowledge and levering the query time response [11]. Our proposal extend previous works and can be used in combination with other techniques for querying performance improvements.

To summarise, existing published IoT ontologies are either complex or domain-specific for sub-domains of IoT. The creation of a lightweight ontology that allows interoperability and discovery of sensory data in heterogeneous platforms

\footnotetext{
${ }^{4}$ http://www.w3.org/2015/spatial/

${ }^{5}$ http://www.fed4fire.eu/

${ }^{6} \mathrm{http}: / /$ vital-iot.eu/

${ }^{7}$ http://www.ict-citypulse.eu/

${ }^{8}$ http://www.openiot.eu/
}

with low complexity and processing time is still an open issue.

\section{IoT-Lite: IoT Modelling AND SEMANTIC ANNOTATION}

While most of the semantic models tend to describe the concepts in great detail and represent various links in IoT systems, we represent only the most used concepts for data analytics in IoT applications, such as sensory data, location and type. See Figure 1 for the model and Figure 3 for an example of an annotated sensor. This paves the way towards creating scalable responsive systems and reduces memory and computational cost of query processing in large scale IoT applications.

In 2003 W3C published a list of sample "Good Ontologies" following specific good practices ${ }^{9}$. The goodness of the ontologies was scored based on five aspects: fully documented; dereferenceable; used by independent data providers; possibly supported by existing tools; and in use by two independent datasets.

IoT-Lite follows these aspects to create a reusable model. We have published the ontology with a web page that fully documents the ontology (aspect 1) with a permanent

\footnotetext{
${ }^{9}$ http://www.w3.org/wiki/Good_Ontologies
} 


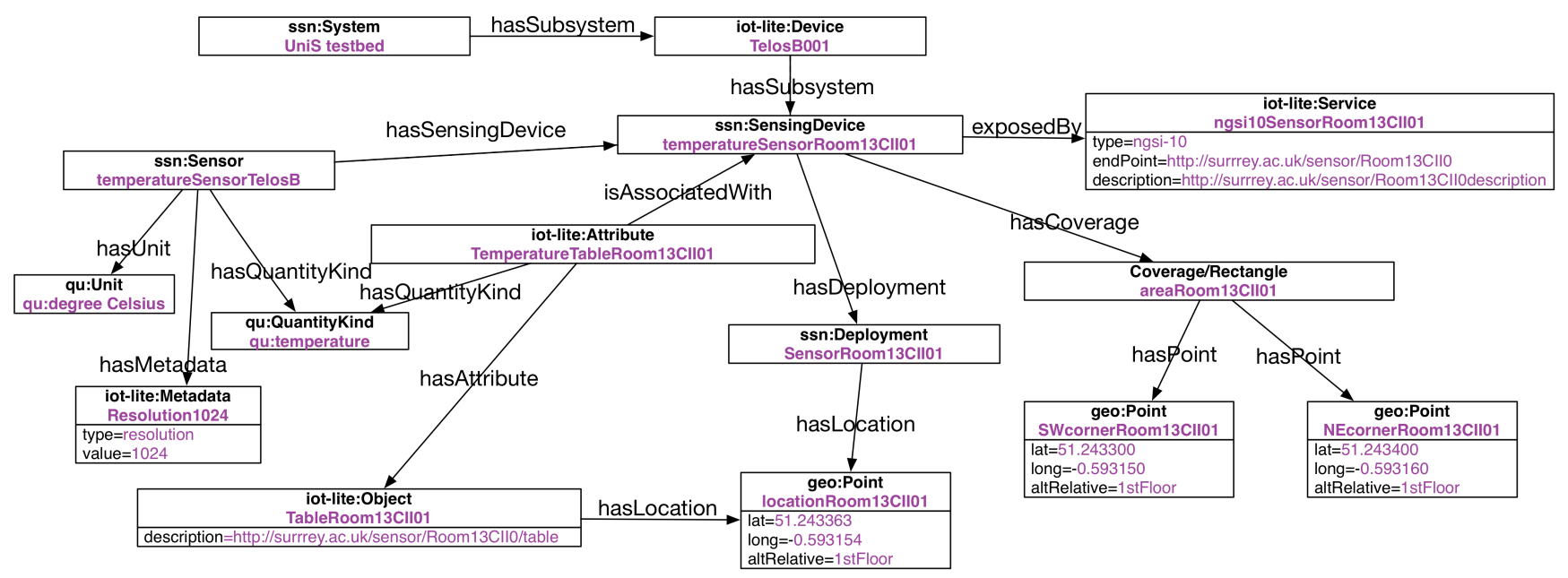

Figure 3: An example of a sensor annotated with the proposed IoT-Lite ontology.

$\operatorname{link}^{10}$, and all the concepts in the ontology are described by a dereferenceable URI (aspect 2). The annotations are applied to IoT testbeds, University of Surrey SmartCampus [6] and SmartSantander [9]. It is planned to be used by other independent platforms in the open calls of the H2020 project FIESTA-IoT ${ }^{11}$ (aspects 3 and 5). We plan to develop annotation and validation tools for IoT-Lite, extending our SAOPY annotation tool ${ }^{12}[5]$ and the SSN validator tool ${ }^{13}$ (aspect 4).

Although the above aspects are essential to create interoperable and reusable ontologies they are not enough to cover scalability, dynamicity and user adoption issues. We propose a set of guidelines for developing scalable ontologies.

1) Design for large-scale.

2) Think of who will use the semantics and design for their neeeds.

3) Provide means to update and change the semantic annotations.

4) Create tools for validation and interoperability testing.

5) Create taxonomies and vocabularies.

6) Re-use existing models.

7) Link data and descriptions to other existing resources.

8) Define rules and/or best practices for providing the values for each property.

9) Keep it simple.

10) Create effective methods, tools and APIs to handle and process the semantics.

In the design of IoT-Lite we have followed these rules. We have designed a lightweight ontology considering the scalability (following rule 1) and will provide tools for

\footnotetext{
${ }^{10}$ purl.oclc.org/NET/UNIS/fiware/iot-lite

${ }^{11} \mathrm{http}: / /$ fiesta-iot.eu

${ }^{12} \mathrm{http} / / /$ iot.ee.surrey.ac.uk/citypulse/ontologies/sao/saopy.html

${ }^{13}$ http://iot.ee.surrey.ac.uk/SSNValidation/
}

annotation and validation (rules 3 and 4), as well as APIs and using existing tools for querying and information processing (rule 10) as we mention previously in this Section. Semantics are only one part of the solution and often not the endproduct. Query methods, machine learning, reasoning and data analysis techniques and methods should be able to effectively use these semantics.

We have designed IoT-Lite (see Figure 1) with a clear purpose of defining only the most-used terms when searching for IoT concepts in the context of data analytics. We studied the most common uses of IoT ontologies (following rule 2) based on our experience with other IoT ontologies used by applications for data analytics. For example, an application that provides the temperature on the move, will query the ontology for the temperature sensor service endpoint at each particular location. The ontology needs the concept of sensor, the quality it measures (temperature) coverage and endpoint. Other concepts are irrelevant in that query. The ontology needs also to have these concepts easily accessible, avoiding deep and distantly-connected terms of the ontology that need complex queries to retrieve the desired results. Therefore the simplicity of the ontology is essential (rule 9). The widely used semantic descriptions on the Web are simple ones such as FOAF.

Another important aspect of semantic models is the interoperability. In the design of IoT-Lite we followed the linked data guidelines ${ }^{14}$. Our ontology is linked with other ontologies (rule 6 and 7). We chose well-known and widely used ontologies, expecting their publications to be stable (e.g. SWEET and SSN). We avoid links to uncommonly used ontologies in order to prevent inconsistencies in case of unexpected deletion of the linked ontologies. In the context of interoperability it is also important to use the

\footnotetext{
${ }^{14}$ http://linkeddata.org/
} 
same vocabulary to be able to share and combine data from different sources. In that sense, we have created a taxonomy of quantity kinds and units which is published on the ontology webpage and is a compilation of terms used in well-known ontologies such as $\mathrm{qu}^{15}$ and qudt ${ }^{16}$ (rule 5 and 7). IoT-Lite is published with a webpage which fully explains the terms used and provides examples (rule 8). This allows reuse and linking with other ontologies.

With the above prerequisites we have created an instantiation of SSN, which is considered the de facto standard of sensor networks ontologies. SSN is not designed to be necessarily used as it is in full form; it is a template to be extended and instantiated. We have customised SSN to make a lightweight ontology with the main concepts being the three well-accepted items in the classification of IoT entities [13]: Entities or objects; resources or devices; and services, namely iot-lite:Object, ssn:Device and iot-lite:Service. Figure 1 shows an overview of the proposed information model. These three concepts are the core concepts of the ontology and are necessary in any ontology describing IoT.

The interrelations between these three concepts are also well-known relationships, that is, an object (or entity) iot-lite:Object has an attribute iot-lite:Attribute which is associated with a device (or resource) iot-lite:Device, which is exposed by a service iot-lite:Service. We built the rest of the ontology around these three main concepts adding the necessary concepts and relationships to provide responses to the standard queries.

To allow the queries to be lighter we have linked most of the concepts of the ontology under one main class (Device) and leave the other two classes lighter. We have spotted at least three main classes of Devices (ssn:Sensor, iot-lite:Actuator, iot-lite:Tag) that we need to separate due to the differences that applications can query for. For example, an application that needs to know the temperature will query for sensors, whilst if the application needs to switch on the lights it will query for actuators. ssn:SensingDevice is directly linked via properties or via inheritance of the relevant properties to the concepts qu:QuantityKind, qu: Units and iot-lite:Coverage. Therefore, we need only three triples to link each sensing device with these concepts (e.g. Sensor1 hasQuantityKind temperature).

In order to allow a common vocabulary to interoperate between different systems we need a taxonomy to describe the measurements of the devices in terms of the quantity kinds and units, such as temperature and degrees Celsius. We have created this taxonomy using individuals from well-

\footnotetext{
${ }^{15}$ www.w3.org/2005/Incubator/ssn/ssnx/qu/qu-rec20.html

${ }^{16} \mathrm{http}: / /$ www.qudt.org/qudt/owl/1.0.0/quantity/
}

know ontologies, such as qu-rec20 ${ }^{17}$ and qudt ${ }^{18}$.

The spatial dimension of the ontology is addressed with the geo ontology ${ }^{19}$ based on WGS84 location coordinates ${ }^{20}$. This simple ontology is widely used and there are some available tools for discovery whether a point belongs to an area, (such our coverage areas circle, rectangle and polygon), and extensions of SPARQL to deal with geolocations. We have added relative locations to these geolocations to annotate locations such as a building or a floor in indoor scenarios, where the geolocation is less intuitive. The relative location also supports linking to resources such as GeoNames $^{21}$ that are publicly available as part of the Linked Open Data cloud ${ }^{22}$.

\section{USE-CASE}

One of the key issues in heterogeneous IoT ecosystems is accessing sensor data from different systems. Enabling a lightweight description of sensors to efficiently manage annotation and discovery of sensor data is essential. In this section, we exemplify the use of the proposed information model, IoT-Lite, using sensor information from the Surrey testbed [7] developed within the EU FP7 project Smart Santander ${ }^{23}$. The testbed consists of 200 IoT nodes/devices provided with 6 sensors each that measure temperature, microphone, vibration, light, presence and energy consumption.

Figure 3 illustrates a sample describing the outcome of one of the temperature sensors in the testbed using the IoTLite ontology. This sensor is associated with the temperature of a room. In this example it can be seen that a table located in Room CII01 has an attribute, temperature, which is associated with the temperature sensor located in the same room. The temperature sensor has a coverage that covers the rectangle of the room, defined by two points in the diagonal corners; measures the temperature with degrees Celsius and a resolution of 1024; and is exposed by a service with endpoint http://surrey.ac.uk/sensor/roomCII01. We have used the geolocation to annotate the latitude and the longitude coordinates. However, we have also annotated the relative altitude as floor1 for better human understanding. Listing 1 is an excerpt of the same temperature sensor annotation in a turtle format.

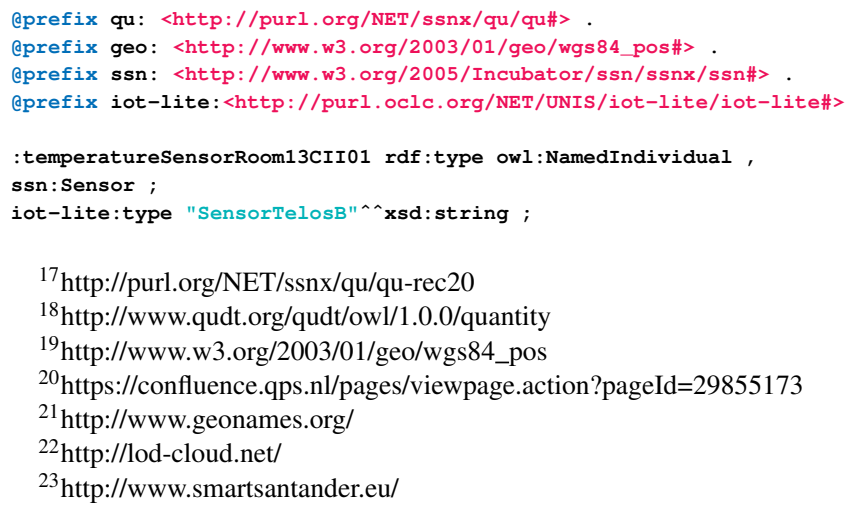




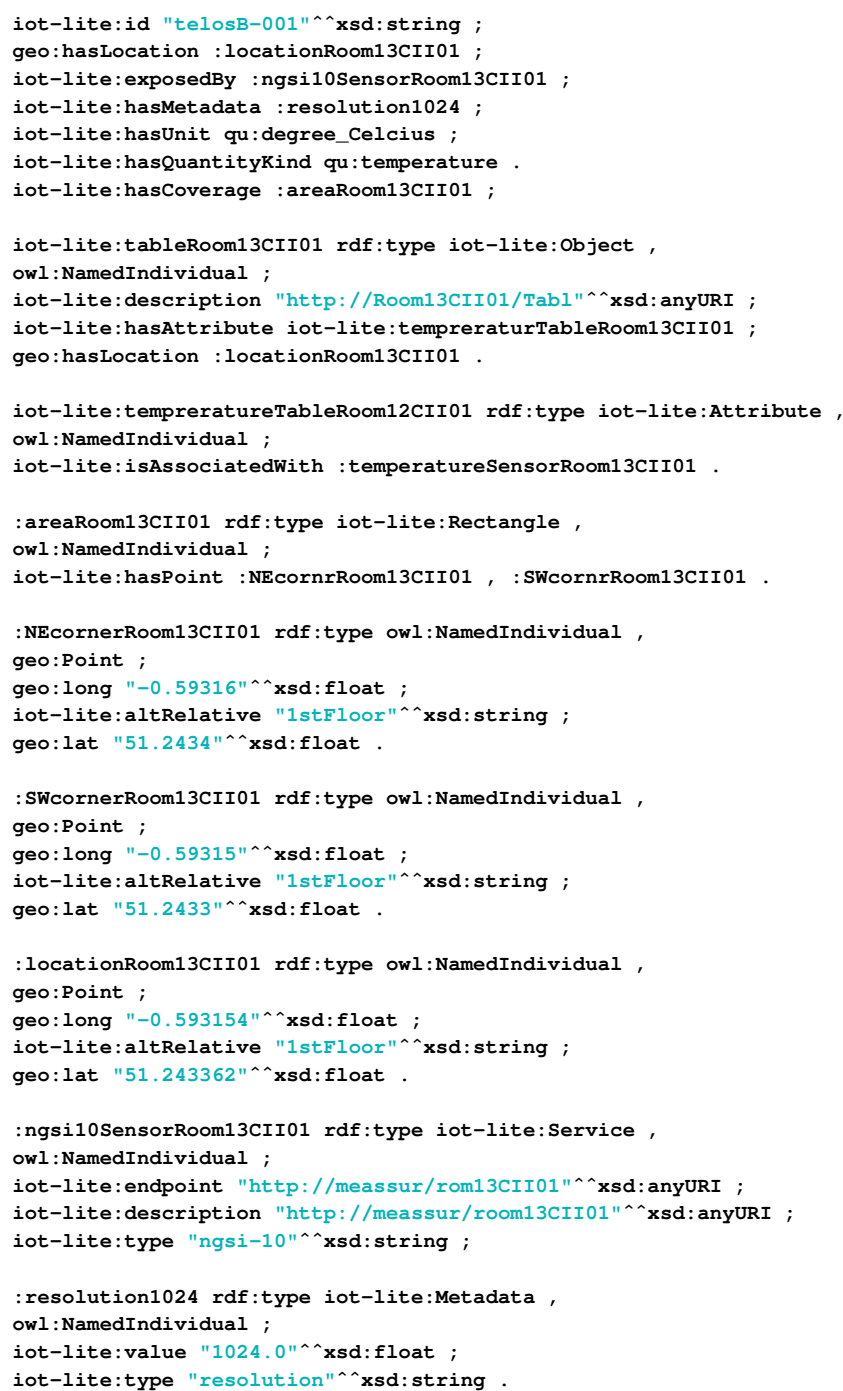

Listing 1: An excerpt from a sensor annotation based on IoT-Lite Ontology.

\section{Evaluations}

In order to validate the scalability and applicability of IoT-Lite we performed some experiments using sensory data from the University of Surrey's SmartCampus testbed. A web application developed in Java was used to annotate the ontology individuals that represent the sensing devices and to store them in a set of Jena TDB triple stores ${ }^{24}$, one for each dataset. We used a Personal Computer (PC) running Windows 7 (x64) operating system with a processor Intel(R) Core(TM) i5-3470 CPU @ 3.20GHz 8GB RAM to act as a server that hosts the web application. We sent remote queries from a different PC located in another subnet. The aim of this experiment was to measure the response time of a common query. With IoT-Lite a common query is defined as

\footnotetext{
${ }^{24}$ https://jena.apache.org/documentation/tdb/
}

in Figure 2 as a query asking for the endpoint of the services that provide the temperature in a particular area. As can be seen, the query is simple. It contains just six triples due to the shallow depth of the IoT-Lite ontology.

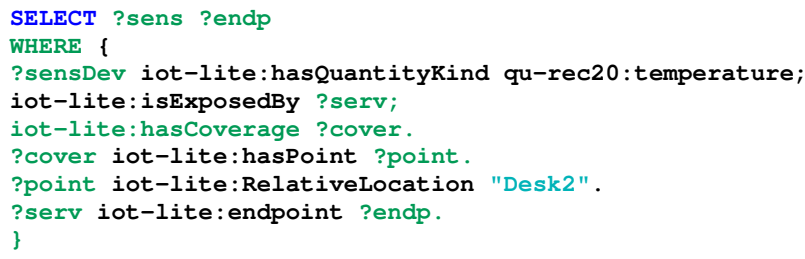

Listing 2: Query performed in the experiments.

We performed this query over different datasets. For that purpose we created four datasets containing 200, 1.000, 10.000 and 100.000 sensors each. The IoT-Lite ontology contains 116 triples by itself. When annotating sensors, each new sensor needs just six triples, and in total the number of triples in each data set are shown in table I.

\begin{tabular}{|l|l|l|l|l|}
\hline datasets: number of sensors & 200 & 1.000 & 10.000 & 100.000 \\
\hline number of triples in IoT-Lite & 1486 & 6926 & 68126 & 680126 \\
\hline number of triples in IoT-A & 1866 & 7946 & 76346 & 760346 \\
\hline
\end{tabular}

Table I: Number of triples in each dataset

To compare the ontology against other solutions we performed the same experiments with IoT-A, another instantiation of SSN aiming to define the architecture of IoT. We chose IoT-A because we have used the IoT-A ontology in one of our components, a discovery element for IoT entities. With this ontology we experienced some of the problems mentioned in the introduction and this motivated us to develop IoT-Lite to replace IoT-A in the discovery component. Figure 2 shows IoT-A. We queried IoT-A with a similar query to that for IoT-Lite, but in this case we needed ten triples to obtain the same results, i.e. the endpoints of services that provide the temperature in a particular area. The IoT-A ontology contains 346 triples by itself. The total number of triples of each data set are also shown in table I.

In order to avoid false perceptions of the round time trip (RTT) due to jitter, we sent the query ten times to each dataset. Figure 4 shows the boxplot results of these 10 queries for each dataset. We can see that the RTT of the query/response is acceptable for every dataset in IoTLite. Even when the dataset contains 100.000 individuals the mean of the RRT is below 200 milliseconds. We can also see that the time of the RTT is less in IoT-Lite than in IoT-A in all the cases, and particularly in large datasets, such as 100.000 sensors, the time of IoT-A is more than doubling the time of IoT-Lite. IoT-Lite performs better than Iot-A for large scale annotations of sensors. 


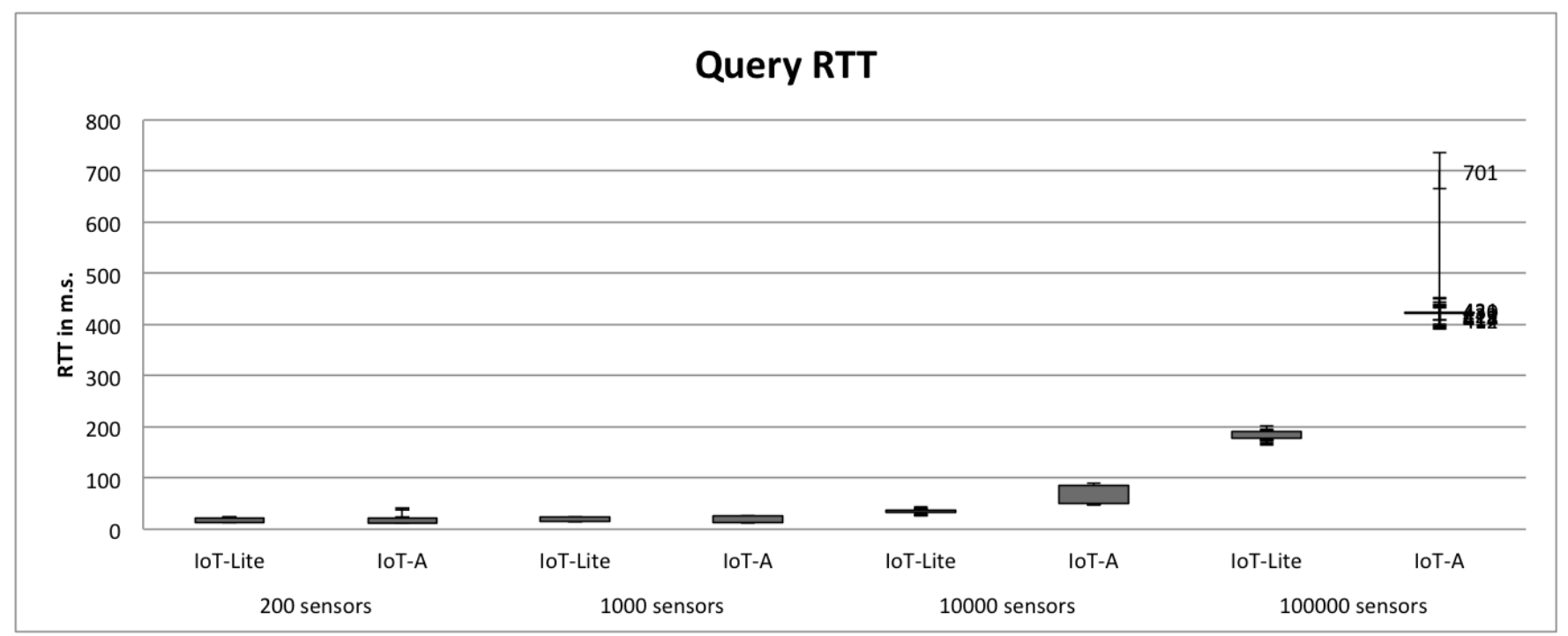

Figure 4: Boxplot of the Round Time Trip (RTT) of the queries required to retrieve the endpoint of a temperature sensor in a certain location depending on the size of the triplestore with both ontologies IoT-Lite ans IoT-A.

\section{CONCLUSIONS}

In this study we proposed a lightweight semantic IoT model, IoT-Lite. The model is an instantiation of SSN with shallow depth, appropriate for real time sensor discovery. We have proposed and followed a set of ontology design guidelines for dynamic and responsive environments. We have demonstrated that the annotation of new sensors in IoT-Lite requires only 6 triples, and that the RTT of a queryresponse is in the range of milliseconds, even for large datasets. We have also assessed our proposal against another instantiation of SSN, IoT-A, and we have demonstrated that IoT-Lite performs better than Iot-A, in terms of memory requirements, computational time and RTT for a queryresponse, reducing the time by half for large datasets, such as for 100.000 sensors. Further work will provide IoT-Lite tools for annotation and validation, similar to SAOPY ${ }^{25}$ and SSN validator $^{26}$. We will also use the IoT-Lite based descriptions to provide interoperability in developing IoT and smart city applications and services.

\section{ACKNOWLEDGEMENT}

The research leading to these results has received funding from the European Commission's in the Seventh Framework Programme for the FIWARE project under grant agreement no. 632893 and in the H2020 for FIESTA-IoT project under grant agreement no. CNECT-ICT-643943.

\section{REFERENCES}

[1] Mike Botts, George Percivall, Carl Reed, and John Davidson. $\mathrm{Ogc} \cap$ sensor web enablement: Overview and high level ar-

\footnotetext{
${ }^{25} \mathrm{http} / / /$ iot.ee.surrey.ac.uk/citypulse/ontologies/sao/saopy.html
}

${ }^{26} \mathrm{http} / / /$ iot.ee.surrey.ac.uk/SSNValidation/ chitecture. In GeoSensor networks, pages 175-190. Springer, 2008.

[2] Michael Compton, Payam Barnaghi, Luis Bermudez, Raul García-Castro, Oscar Corcho, Simon Cox, John Graybeal, Manfred Hauswirth, Cory Henson, Arthur Herzog, et al. The SSN ontology of the W3C semantic sensor network incubator group. Web Semantics: Science, Services and Agents on the World Wide Web, 17:25-32, 2012.

[3] Suparna De, Tarek Elsaleh, Payam Barnaghi, and Stefan Meissner. An internet of things platform for real-world and digital objects. Scalable Computing: Practice and Experience, 13(1), 2012.

[4] Cory A. Henson, Josh K. Pschorr, Amit P. Sheth, and Krishnaprasad Thirunarayan. Semsos: Semantic sensor observation service. In Collaborative Technologies and Systems, 2009. CTS'09. International Symposium on, pages 44-53. IEEE, 2009.

[5] Sefki Kolozali, Maria Bermudez-Edo, Daniel Puschmann, Frieder Ganz, and Payam Barnaghi. A knowledge-based approach for real-time iot data stream annotation and processing. In Internet of Things (iThings), 2014 IEEE International Conference on, and Green Computing and Communications (GreenCom), IEEE and Cyber, Physical and Social Computing (CPSCom), IEEE, pages 215-222. IEEE, 2014.

[6] Michele Nati, Alexander Gluhak, Hamidreza Abangar, and William Headley. Smartcampus: A user-centric testbed for internet of things experimentation. In Wireless Personal Multimedia Communications (WPMC), 2013 16th International Symposium on, pages 1-6. IEEE, 2013.

[7] Michele Nati, Alexander Gluhak, Jaroslaw Domaszewicz, Spyros Lalis, and Klaus Moessner. Lessons from smartcampus: External experimenting with user-centric internetof-things testbed. Wireless Personal Communications, pages $1-15,2014$. 
[8] OneM2M. Study of abstraction and semantics enablement v.0.7.0. study of existing abstraction and semantic capability enablement technologies for consideration by onem $2 \mathrm{~m}$. Technical Report OneM2M, (TR 0007), 2014.

[9] Luis Sanchez, Luis Muñoz, Jose Antonio Galache, Pablo Sotres, Juan R Santana, Veronica Gutierrez, Rajiv Ramdhany, Alex Gluhak, Srdjan Krco, Evangelos Theodoridis, et al. Smartsantander: IoT experimentation over a smart city testbed. Computer Networks, 61:217-238, 2014.

[10] Amit Sheth, Cory Henson, and Satya S Sahoo. Semantic sensor web. Internet Computing, IEEE, 12(4):78-83, 2008.

[11] Markus Stocker, Narasinha Shurpali, Kerry Taylor, George Burba, Mauno Rönkkö, and Mikko Kolehmainen. Emrooz: A scalable database for SSN observations. In Joint Proceedings of the 1st Joint International Workshop on Semantic Sensor Networks and Terra Cognita (SSN-TC 2015) and the 4th International Workshop on Ordering and Reasoning (OrdRing 2015) co-located with the 14th International Semantic Web Conference (ISWC 2015), Bethlehem, Pennsylvania, United States, October 11th - and - 12th, 2015., pages 1-12, 2015.

[12] Kerry Taylor and Ed Parsons. Where is everywhere: bringing location to the Web. IEEE Internet Computing, 19(2):83-87, March/April 2015.

[13] Ovidiu Vermesan, Peter Friess, Patrick Guillemin, Sergio Gusmeroli, Harald Sundmaeker, Alessandro Bassi, Ignacio Soler Jubert, Margaretha Mazura, Mark Harrison, M Eisenhauer, et al. Internet of things strategic research roadmap. O. Vermesan, P. Friess, P. Guillemin, S. Gusmeroli, H. Sundmaeker, A. Bassi, et al., Internet of Things: Global Technological and Societal Trends, 1:9-52, 2011.

[14] Wei Wang, Suparna De, Ralf Toenjes, Eike Reetz, and Klaus Moessner. A comprehensive ontology for knowledge representation in the internet of things. In Trust, Security and Privacy in Computing and Communications (TrustCom), 2012 IEEE 11th International Conference on, pages 1793-1798. IEEE, 2012. 